\title{
SOBRE LA ENSEÑANZA DE LA POESÍA LATINA EN LA ALTA EDAD MEDIA: EL CAPÍTULO 26 DE LOS CASUS SANCTI GALLI Y SU CONTRIBUCIÓN AL ESTUDIO DEL CURSUS RHYTHMICUS
}

\author{
Lorenzo MARTINEZ ANGEL \\ Domno Iosepho sapienti et docto Torne
}

La famosa crónica monástica medieval titulada Casus Sancti Galli abunda en informaciones de tipo cultural de muy diverso ámbito, propor-cionándolas en un grado considerablemente más elevado que las crónicas hispanas. No es casual, pues es sobradamente conocida la importancia que en diversos campos del saber tuvo la abadía de San Galo1.

Nos centramos, en esta ocasión², en un pasaje, recogido en el capítulo 26, en el que se relata una visita que el abad-obispo (de Constanza) Salomón (890-919) realizó a las aulas del monasterio ${ }^{3}$. Además de las substanciosas noticias sobre las costumbres escolares de la abadía, lo que nos interesa destacar es la existencia de tres niveles en la enseñanza de la lengua latina que los jóvenes estudiantes recibían: "Cum ille subiunxisset: "Quomodo?", parvuli Latine pro nosse, medii rithmice, ceteri vero metrice, quasi pro rostris rhetorice etiam illum [al abad-obispo Salomón] affantur."

Así pues, había tres niveles: el de los pequeños que comenzaban su conocimiento del latín, los que se ejercitaban en el cursus rythmicus y los que ya componían poesía métrica al modo clásico, lo cual supone una evolución muy lógica de la enseñanza. Esto, ya de por sí, es algo de gran interés tanto desde el punto de vista de la Historia de la Educación como el de la Filología. Por lo que respecta a lo primero, ilumina un aspecto de la mejor enseñanza medieval que indica una práctica que probablemente no se limitase sólo a la abadía sangalense sino a otros centros educativos de la época. Y, por lo que afecta a la Filología, es de particular importancia con respecto al cursus rhythmicus, puesto que se ha indicado que su uso hasta finales del siglo VII, resurgiendo desde en tiempos plenomedievales 4 : aunque su uso hubiese mermado, este testimonio de San Galo permite comprobar cómo su enseñanza y utilización se mantenía.

\footnotetext{
${ }^{1} \mathrm{Su}$ destacado papel cultural llega a extremos tan curiosos como ser el lugar donde primeramente se documenta la palabra scriptorium: "El término latino scriptorium no había entrado en uso alguno antes de la época carolingia, y así lo desconoce totalmente Isidoro de Sevilla. Sin emplear el vocablo (sin duda creado en el siglo IX por analogía con dormitorium, refectorium, ambulatorium), hacia 800 diseña las funciones que allí se cumplen Alcuíno de York. Usa el vocablo por primera vez unos doscientos años más tarde Ekkerhard (IV) de San Galo, donde quizá se originó y conservó, porque a aquella abadía también remite su uso, hacia finales del siglo IX, en una preciosa Oración en el escriptorio. Sólo después, hacia el siglo XII, el término se vulgarizó." (Manuel C. Díaz y Díaz, El escriptorio de Valeránica: Codex Biblicus Legionensis. Veinte estudios, León 1991, 5372, concretamente p. 53). De hecho, el testimonio que aduce el Prof. Díaz y Díaz procede de los Casus Sancti Galli.

${ }^{2}$ En alguna otra nos hemos ocupado ya de temas recogidos en esta crónica (Más referencias etnográficas en textos medievales: el capitulo 43 de los Casus Sancti Galli: Revista de Folklore 257 (2002) 161-163).

${ }^{3}$ Remitimos al lector interesado al texto de la crónica. Hemos consultado la siguiente edición: Ekkerhardi IV, Casus Sancti Galli (editionis textum paravit Hans F. Haefele), Darmstadt 1991. El texto latino del capítulo 26 se encuentra en las páginas 64 y 66. También recoge la anécdota, e incluye una muy parcial traducción del episodio fray Justo Pérez de Urbel, Las grandes abadías benedictinas, Madrid, c. 1928, 323-324.

${ }^{4}$ Sebastián Mariner Bigorra, Peculiaridades estilísticas de las obras de Santo Martino: Santo Martino de León. Ponencias del I Congreso Internacional sobre Santo Martino en el VIII Centenario de su obra literaria 1185-1985, León 1987, 459-492, concretamente 485, nota 56, citado a J. Guillén, "Origen y constitución del "cursus rítmico", Helmantica 41-42, 1962, 309-350. Escribe así D. Sebastián Mariner citando a J. Guillén: “...señala la vigencia del cursus hasta fines del s. VII (en cuyo primer tercio había escrito S. Isidoro) y su renacimiento a partir de finales del s. XI (en que conmemoramos la redacción de la obra de Sto. Martino)". Debe haber una errata de imprenta, pues Santo Martino comenzó su obra a finales del siglo XII.
} 
Pero, además, la crónica que nos ocupa nos permite conocer algunos de los versos que los estudiantes dijeron al abad-obispo Salomón:

"Quorum duorum, quoniam a patribus verba recepimis, unus:

"Quid", inquit, "tibi fecimus tale, ut nobis facias male?"

"Appellamus regem, quia nostram facimus legem."

At alter versificator inquit:

"Non nobis, piae, spes fuerat, cum sis novus hospes

Ut vetus impeius trasvertere tute velis ius."

A continuación analizaremos los que Fray Justo Pérez de Urbel calificó como 'malos versos' 5 . El primer versificador utilizó el cursus rythmicus -lo que más nos interesa en este trabajo-; el segundo, la métrica clásica.

Analizando los dos primeros versos rítmicos, vemos, además del uso de rima interna, la utilización del cursus planus, con acento en la segunda y quinta sílabas comenzando por el final de la cláusula. En el primer verso: fécimus tále / fácias málé.

El segundo es más complejo, pues sigue la rima interna, pero ya no es tan regular en el uso del cursus planus, que sí aparece en fécimus légem, aunque no en appellámus régem. Para explicar esto podemos pensar en varias posibilidades. La primera es que el estudiante no consiguió hacerlo bien, lo cual se podría ver respaldado por el lenguaje sencillo que utiliza y por tratarse de una improvisación.

Pero hay otra posible, y estaría apoyada por algún tipo de 'tradición'7. Decimos esto porque appellámus régem, con acento 2-4, es, no métrica sino rítmicamente, un ditroqueo; los ditroqueos "realmente no debería ser considerados cláusulas" 8 . Y, sin embargo, en poemas rítmicos de Valerio del Nierzo se da la presencia, junto a versos con diversos tipos de cursus -la mayoría-, de versos con ditroqueos, etc. ${ }^{9}$

Continuando nuestro análisis, nos fijaremos ahora en la corrección del uso del cursus planus en cuanto a la elección de la extensión de las palabras que lo componen, pues, como es sabido: "La última palabra de cada una de las especies del cursus es, preferentemente, en el cursus planus, una dicción de tres sílabas con el acento en la penúltima precedida de otra palabra con el acento también en la penúltima."10

En el caso que nos ocupa del estudiante de San Galo es evidente que esto no se respeta. ¿Es normal? La respuesta es afirmativa, y se comprueba en diversos momentos de la Edad Media. Así, en poemas rítmicos del ya citado Valerio del Bierzo (de finales del siglo VII) encontramos ejemplos de cursus planus como flébile cármen o contrítio crébra11. Y a caballo entre los siglos XII y XIII Santo Martino de León escribe ejemplos de

\footnotetext{
${ }^{5}$ Fray Justo Pérez de Urbel, o. c., 324.

${ }^{6}$ Marcamos con tildes el acento para mayor claridad expositiva.

${ }^{7}$ Hay que tener en cuenta lo siguiente: "A cuatro clases podemos reducir las combinaciones del ritmo medieval, si bien autor hay que señala hasta dieciséis especies diferentes; y es de notar para fijar el número de sílabas, que entre ellas tienen cabida, casi todas las licencias poéticas. Como nos encontramos ante una materia poco estudiada aún, no existe uniformidad entre los escasos autores modernos que de ella se ocupan". (Antonio Viñayo, "El cursus rythmicus en el Prólogo de las obras de Santo Martino", Studium Legionense 16, 1975, 313-329, concretamente p.326).

${ }^{8}$ Manuel C. Díaz y Díaz, Anecdota wisigothica I. Estudios y ediciones de textos literarios menores de época visigoda, Salamanca 1958, p.100.

${ }^{9}$ ID., ibid., 100-101.

${ }^{10}$ Antonio Viñayo, "El crítico leonés Antonio de Valbuena y la oración del Santísimo Sacramento", Studium Legionense 7, 1966, 161-175, aquí la p.169.

${ }^{11}$ Manuel C. Díaz y Díaz, Anecdota wisigothica..., p. 105.
} 
cursus planus como glóriam dédit o cónsequi póssit ${ }^{12}$.

El uso del cursus rythmicus en San Galo se constata también en otros lugares de los Casus Sancti Galli; así, en el capítulo 45 leemos un versos, con uso, también, del cursus planus:

hoc pánthema pía (2-5) / celaverat ípsa María (2-5)13.

Con respecto a los versos del otro alumno versificador, pretenden ser hexámetros, aunque lo consigue mejor en el segundo que en el primero. Pero aquí quizá haya que pensar, por razones lingüísticas, en la impericia del autor como causa ${ }^{14}$. No obstante, la gran calidad de los versos métricos de diversos monjes escritores medievales de San Galo queda fuera de duda, y no es éste el lugar para citar los muchos ejemplos surgidos de la pluma de destacados monjes escritores de la abadía que nos ocupa o los numerosos estudios que han merecido ${ }^{15}$.

El análisis del cursus rhythmicus seguirá proporcionándonos, sin duda, datos de interés para comprender mejor la enseñanza, la literatura y, en general, la cultura latina de la Edad Media occidental.

\footnotetext{
${ }^{12}$ Antonio Trobajo Díaz, La calidad literaria del "Sermo in Assumptione" de Sto. Martino: Santo Martino de León. Ponencias..., 493-512, concretamente pp. 504-505. En la pág. 504 se lee: "Nótese, no obstante, que a estas alturas del Medioevo no se puede exigir la puridad de las normas, que fallarán principalemente por la selección de la última palabra en cuanto a su número de sílabas." Si aparecen estas variaciones en los siglos VII y IX-X, parece claro que la causa de que existan éstas en las obras de Santo Martino no se debe a la fecha en las que se escribieron. Más acertadas son las palabras de Antonio Viñayo cuando escribe: "Los ejemplos señalados anteriormente llenan todas estas exigencias y están tomados del prólogo de las Obras de Santo Martino. Pero advierten los tratadistas no ser necesaria esta rigidez en la elección de las palabras, y basta que los acentos recaigan en los lugares señalados para cada uno de los cursus, aunque las sílabas se repartan de otro modo en las palabras finales." (Antonio Viñayo, El cursus rythmicus..., p. 327).

${ }^{13}$ En ipsa María se respeta la citada regla del cursus planus de terminar en palabra trisílaba con acento en la penúltima precedida por otra palabra también llana.

${ }^{14}$ Sobre las características de la métrica latina medieval remitimos a diversos trabajos aparecidos en Actas. I Congreso Nacional de Latín Medieval, Madrid 1995. Respecto a la enseñanza de la métrica clásica en la Edad Media téngase en cuenta que "para la correcta versificación latina se aprendía la poesía en libros de Mallio Teodoro, de Diomedes y de Beda el Venerable (De arte metrica)" (Peter Ochsenbein, Enseñar y aprender en el monasterio de Sankt Gallen, en Werner Vogler [dir.], La cultura de la abadía de Sankt Gallen, Madrid 1992, 133-148, concretamente p. 138).

${ }^{15}$ No queremos dejar de hacer mención del joven Burcardo, que con sus hexámetros improvisados consiguió que Heduvigis le enseñase griego, episodio que recogen los Casus Sancti Galli (capítulo 94) y que llamó la atención, p. ej., de Fray Justo Pérez de Urbel (o. c., 179-181). Sobre la cultura de San Galo remitimos a la obra dirigida por Werner Vogler citada en la nota anterior, donde se contienen abundantes referencias bibliográficas, destacando al respecto de la producción escrita de la abadía que nos ocupa el capítulo de Walter Berschin titulado Literatura latina en Sankt Gallen (pp. 145-156).
} 